\title{
Reference fallible endgame play
}

\author{
Article
}

Published Version

Haworth, G. M. (2003) Reference fallible endgame play. ICGA Journal, 26 (2). pp. 81-91. ISSN 1389-6911 Available at https://centaur.reading.ac.uk/4550/

It is advisable to refer to the publisher's version if you intend to cite from the work. See Guidance on citing.

Publisher: The International Computer Games Association

All outputs in CentAUR are protected by Intellectual Property Rights law, including copyright law. Copyright and IPR is retained by the creators or other copyright holders. Terms and conditions for use of this material are defined in the End User Agreement.

\section{www.reading.ac.uk/centaur}

\section{CentAUR}

Central Archive at the University of Reading

Reading's research outputs online 


\title{
REFERENCE FALLIBLE ENDGAME PLAY
}

\author{
G.M C. Haworth ${ }^{l}$ \\ Reading, UK
}

\begin{abstract}
A reference model of fallible endgame play is defined in terms of a spectrum of endgame players whose play ranges in competence from the optimal to the anti-optimal choice of move. They may be used as suitably skilled practice partners, to assess a player, to differentiate between otherwise equi-optimal moves, to promote or expedite a game result, to run Monte-Carlo simulations, and to identify the difficulty of a position or a whole endgame.
\end{abstract}

\section{INTRODUCTION}

When a player is armed with perfect information from endgame tables, EGTs, questions still arise as to how to play a fallible opponent. In a drawn position, how best can the attacker pressure the defender or the defender resist the attacker (Levy 1987, 1991; Nunn, 2002; Schaeffer 1991, 1997)? How can the opponent be given the best opportunity to concede depth in a situation where a draw claim is possible (Haworth, 2000, 2001)? How can a player identify and adapt to apparent opponent fallibility? More generally, one might ask how well a fallible player is likely to perform in an endgame, and how 'difficult' that endgame or a specific position is. To address these questions, a model of fallible endgame play is defined here. It is a spectrum of Reference Endgame Players, REPs, with a linear range of competence ranging from metric-optimal play via the random player to anti-optimal play. REPs with greater skill are more likely to play better rather than worse moves.

Section 2 describes an endgame as a finite-state system while Section 3 defines the requirement for a spectrum of REPs. Section 4 defines properties of the REP Preference Function which ensure that the set of REPs $\left\{R_{c}\right\}$ is also a spectrum, and then converts preference values to probabilities. Section 5 proves that the properties defined in Section 4 guarantee the spectrum properties of $\left\{R_{c}\right\}$. Section 6 defines the $S_{c}$ function subsequently used here. Section 7 examines basic REP use while Section 8 demonstrates, by revisiting the historic BrowneBELLE KQKR games, how to assess the competence of the opponent. Section 9 surveys ways of exploiting REPs and a fallible opponent. The summary mentions some open questions currently being considered.

\section{FINITE-STATE ENDGAMES}

A specific endgame for which an EGT has been computed can be thought of as a system with a finite $n s$ states. Western Chess, KQKR, and the DTC metric ${ }^{2}$ are the exemplar scenario here. Positions $P$ may be regarded as mathematically, if not chessically, equivalent if and only if they have the same theoretical value val and depth $d$ in some metric. The equivalence classes are the system states: moves effect transition from state to state. The side to move, $\mathrm{stm}$, from its perspective, regards $s_{1} \geq s_{2}$, i.e., $s_{1}$ is no worse than $s_{2}$, if $\operatorname{val}\left(s_{1}\right)>\operatorname{val}\left(s_{2}\right)$ or $\operatorname{val}\left(s_{1}\right)$ $=\operatorname{val}\left(s_{2}\right)$ and the win is no longer or loss no shorter than in state $s_{2}$.

In KQKR, there are 1-0 wins of DTC depth 0 to $n_{W}=31$, draws and $0-1$ wins of DTC depths 0 to $n_{L}=3$. A 'depth 0' win is one in which the winner does not need another move to secure the win and these include stmmated positions, all decisive subgame positions regardless of DTC depth, and the much rarer 'stm forceconverts to loss' positions. The KQKR system therefore has $1+n_{W}+1+n_{L}+1=37$ states, and these may be numbered 0-36 in order of decreasing attractiveness to White or the stm. Thus, e.g., $s_{0-31}$ are 1-0 wins in 0-31 moves, $s_{32}$ is the draw state, and $s_{33-36}$ are 0-1 wins in 3-0 moves. Depth 0 states 0 and 36 are absorber states.

\footnotetext{
${ }^{1}$ 33, Alexandra Rd., Reading, Berkshire, RG1 5PG, UK. Email: guy_haworth@hotmail.com. An earlier version of this paper was presented at the ICCA $7^{\text {th }}$ Computer Olympiad Workshop, Maastricht (2002).

${ }^{2} \mathrm{DTC} \equiv$ Depth to Conversion, i.e., (winner's) moves to change of force and/or mate. DTM(ate) is another metric.
} 


\section{REFERENCE ENDGAME PLAYERS}

An REP $R_{c}$ in position $P$ and state $s$ chooses its move stochastically with a probability function, based on a Preference Function $S_{c}$ which involves only the competence parameter $c$ and the value and depth of successor positions $\left\{P_{\alpha}\right\}$. The $R_{c}$ has mathematical but no other game-specific knowledge and it is worth emphasising that each choice of move is independent of previous choices of move. Metaphorically, REP $R_{c}$ comes to the gaming table with its own 'designer' roulette wheel - having slots of different sizes for the moves, reflecting a preference for better moves. The rolling ball is more likely to lodge in the wider slots representing better moves.

We require that the set $\left\{R_{c}\right\}$ is in fact a linear, ordered spectrum of $R_{c} \mathrm{~s}$ with two properties:

p01: For $R_{0}$, all moves are equally likely. ' $R_{\infty}$ ' $\equiv \lim _{c \rightarrow \infty} R_{c}$ exists ${ }^{3}$ and is the infallible player. ' $R_{-\infty}$ ' $\equiv \lim _{c \rightarrow-\infty} R_{c}$ exists and is the anti-infallible player.

p02: $c 2>c 1 \Rightarrow R_{c 2}$ 's expectations of successor state $\mathrm{E}[s \mid P]$ and value $\mathrm{E}[\operatorname{val}(s) \mid P]$ are no worse than $R_{c l}$ 's.

The $S_{c}$ properties mandated in Section 4 are shown in Section 5 to imply $p 01-p 02$. The infallible player always chooses a best move in terms of the metrics chosen: the anti-infallible player always chooses a worst move.

\section{PREFERENCE FUNCTIONS AND PROBABILITIES}

Let $S_{c}\left(s_{j}\right)$ be the preference of REP $R_{c}$ for a single move $m_{\alpha}$ to state $s_{j} . S_{c}\left(m_{\alpha}\right) \equiv S_{c}\left(s_{j}\right)$ is a function only of the value and depth of $s_{j}$ and this is clearer in the notations $S_{c}\left(\operatorname{val}_{k}, d_{j}\right)$ and $S_{c}\left(\operatorname{val}\left(s_{j}\right), d\left(s_{j}\right)\right)$.

We require $S_{c}\left(s_{j}\right)$ to have the following reasonable properties ( $\uparrow \equiv$ 'increases', $\downarrow \equiv$ 'decreases'):

p03: $S_{c}\left(s_{j}\right)$ is finite and positive: no move has zero or infinite preference.

p04: $S_{0}\left(s_{i}\right)=$ constant. This is equivalent to $p 01$ on $R_{0}$, merely restating that requirement in terms of $S_{0}$.

p05: For some $d_{W}>n_{W}$ and $d_{L}>n_{L}, S_{c}($ draw $)=S_{c}\left(\right.$ win,$\left.d_{W}\right)=S_{c}\left(\right.$ loss,$\left.d_{L}\right)$

q06: $W F_{d}(c)=S_{c}($ win,$d+1) / S_{c}($ win, $d) \downarrow$ as $c \uparrow: \lim _{c \rightarrow \infty} W F_{d}(c)=0$ and $\lim _{c \rightarrow-\infty} 1 / W F_{d}(c)=0$.

q07: $L F_{d}(c)=S_{c}($ loss,$d) / S_{c}($ loss,$d+1) \downarrow$ as $c \uparrow: \lim _{c \rightarrow \infty} L F_{d}(c)=0$ and $\lim _{c \rightarrow-\infty} 1 / L F_{d}(c)=0$.

q08: For $c \neq 0, \operatorname{sign}(c) . S_{c}\left(s_{W}\right) \downarrow$ as depth of 'win' state $d\left(s_{W}\right) \uparrow$.

q09: For $c \neq 0, \operatorname{sign}(c) . S_{c}\left(s_{L}\right) \uparrow$ as depth of 'loss' state $d\left(s_{L}\right) \uparrow$.

p08: For $c>(<) 0, W_{c}(d)=S_{c}($ win,$d) / S_{c}($ win,$d+1) \downarrow(\uparrow)$ as $d \uparrow$ and $\lim _{d \rightarrow \infty} W_{c}(d)=1$.

For $c>(<) 0, L_{c}(d)=S_{c}($ loss,$d+1) / S_{c}($ loss,$d) \downarrow(\uparrow)$ as $d \uparrow$ and $\lim _{d \rightarrow \infty} L_{c}(d)=1$.

We assume that wins in $d$ and $d+1$ are more differentiable than wins in $d+1$ and $d+2$.

The function $S_{c}$ generates preference values for all available moves and these must now be scaled to moveprobabilities. We may also assume that some moves have independently been discounted or weighted.

Let $R_{c}$ in state $s_{i}$, position $P$, have $n_{j}$ moves $m_{\alpha}: P \rightarrow P_{\alpha}, s_{i} \rightarrow s_{j}$. Pre-weighting, $R_{c}$ 's preference for $s_{j}$ is $n_{j} . S_{c}\left(s_{j}\right)$. Weighting move-choices can reflect presumed search depths or strategies, subjective inclination and/or competence on the part of the opponent (Jansen, 1992b, 1993), e.g., "favours checking the King", "tends to play moves with high tactical threat value" and so on. Examples of weighting which may be combined are:

- zero-weight moves conceding the position's theoretical value

- if other moves exist, zero-weight any move to a lost position

- $\quad$ if other moves exist, zero-weight moves conceding theoretical value in $d \leq H$ (orizon) moves ${ }^{4}$

- if there are wins in $d \leq H^{5}$, zero-weight moves which are not metric-optimal

- give weight $v_{\alpha}>1$ to an apparently strong move $m_{\alpha}$; weight $1>v_{\alpha}>0$ to an apparently weak move

Therefore, after such weighting, the $R_{c}$ 's moderated preference for move $m_{\alpha}$ is $v_{\alpha} . S_{c}\left(s_{j}\right)$. Let the weight, rather than the number of moves to state $s_{j}$ be $w_{j}$. The final step is the trivial one of scaling $\Sigma_{\alpha} v_{\alpha} \cdot S_{c}\left(s_{j}\right)=\Sigma_{j} w_{j} . S_{c}\left(s_{j}\right)$ to a sum of one to get the actual probabilities, $\mathrm{P}\left[m_{\alpha}\right]$ and $\mathrm{P}\left[s_{j}\right]$ respectively, of move $m_{\alpha}$ and state $s_{j}$ being chosen.

\footnotetext{
${ }^{3} R_{c}$ tends to a limit in the sense that its move-choice probabilities all converge to limits.

${ }^{4}$ e.g., $H=7$ would reflect an assumption that the player, or opponent, will find wins or losses up to 14 plies deep.

${ }^{5}$ Draws which must be forced in $d$ moves are not discussed here but can be accommodated in this stochastic model.
} 
Let $\Omega_{c}=\Sigma_{j} w_{j} \cdot S_{c}\left(s_{j}\right)$ and let $\omega_{c}=1 / \Omega_{c}$ so that $\omega_{c} \Sigma_{j} w_{j} \cdot S_{c}\left(s_{j}\right)=1$.

Let $T_{j, c}=\omega_{c} \cdot w_{j} \cdot S_{c}\left(s_{j}\right)$, the sum probability of $R_{c}$ moving to state $s_{j}$, with $T_{D, c}$ the lowest-indexed $T_{j, c} \neq 0$. Then: $R_{c}{ }^{\prime}$ 's expected state-number and theoretical value are $\mathrm{E}_{c}[s \mid P] \equiv \sum_{j} j . T_{j, c}$ and $\mathrm{E}_{c}[\operatorname{val}(s) \mid P] \equiv \sum_{j} \operatorname{val}\left(s_{j}\right) \cdot T_{j, c}$.

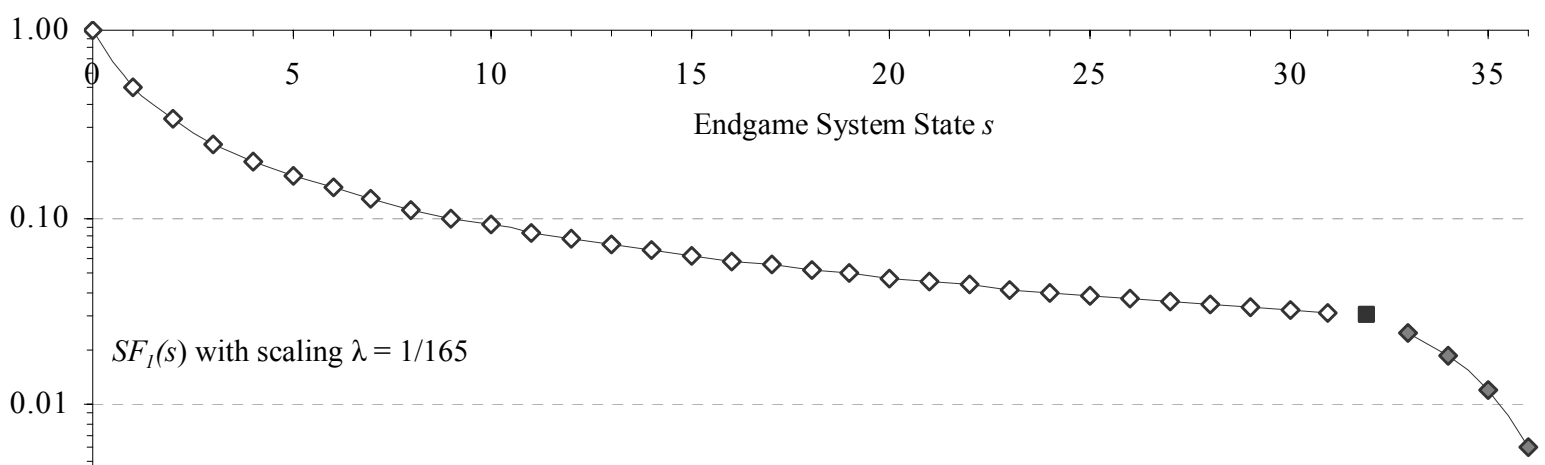

Figure 1: KQKR (DTC) wtm Preference Function $S F_{l}(s), c=\kappa=1, d_{W}=32, d_{L}=4$.

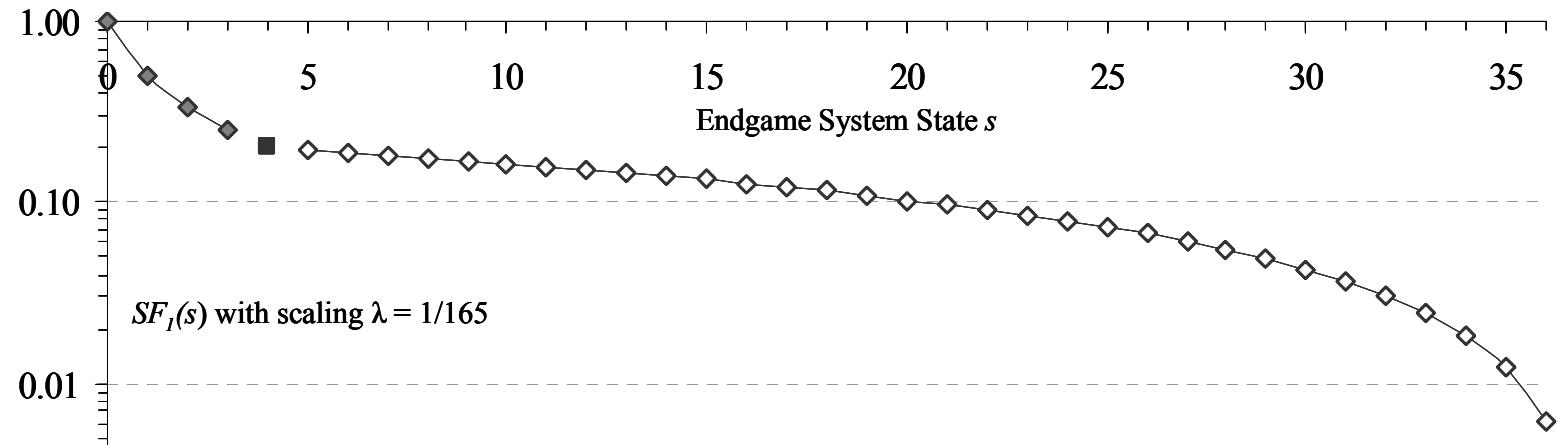

Figure 2: KRKQ (DTC) wtm Preference Function $S F_{l}(s), c=\kappa=1, d_{W}=4, d_{L}=32$.

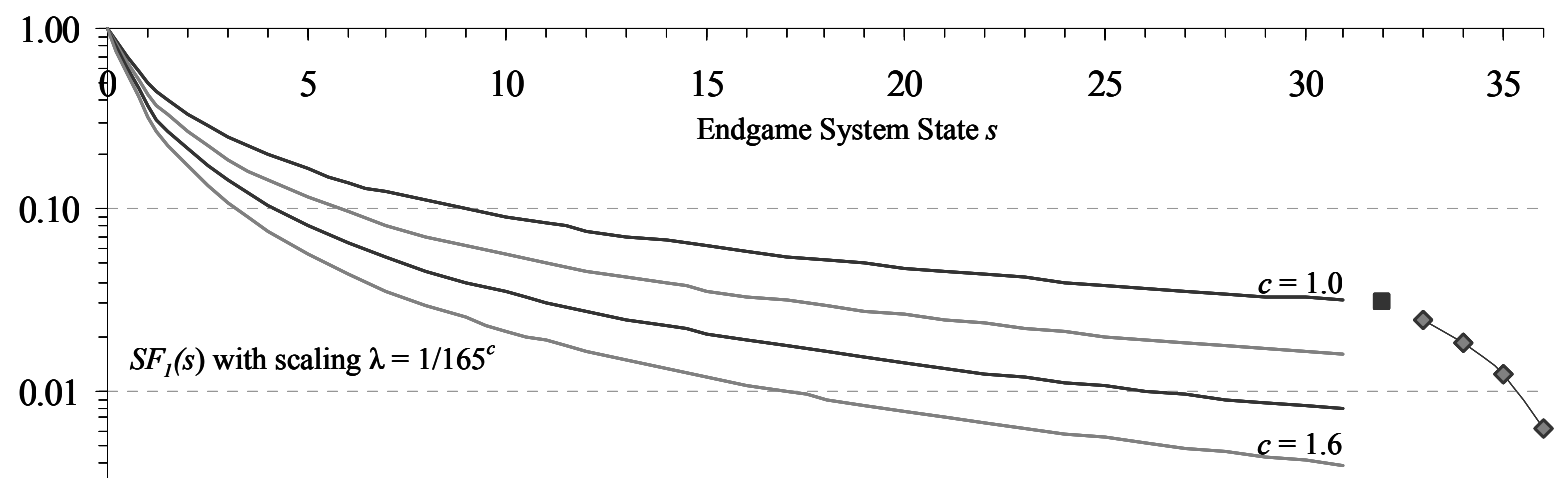

Figure 3: KQKR (DTC) wtm Preference Function $S F_{I}(s), c=1.0-1.6, \kappa=1, d_{W}=32, d_{L}=4$. 


\section{THEOREMS ON REPS AND PREFERENCE FUNCTIONS}

The following theorems are expressed assuming that the states are numbered in order of decreasing attractiveness to the side to move, which, without loss of generality is assumed to be White. The win/draw/loss states are therefore numbered respectively 0 to $n_{W}, n_{W}+1$ and $n_{W}+2$ to $n s-1$. There is no assumption that White is the nominally stronger side, or indeed that White is at least drawing as is conventional in chess studies. However, the behaviour of the R-side $R_{c}$ in the KRKQ of Figure 2 could equally well be represented by renumbering the states $\{0,1, \ldots, n s-1\}$ as $\{n s-1, n s-2, \ldots, 0\}$, effectively reflecting the graph of Figure 2 left to right.

Theorem 1. With the following definitions of properties $p 06-p 07,\{p 03-p 05, q 06-q 09\} \Leftrightarrow\{p 03-p 07\}$ :

p06: $F_{j}(c)=S_{c}\left(s_{j+1}\right) / S_{c}\left(s_{j}\right) \downarrow$ as $c \uparrow: \lim _{c \rightarrow \infty} F_{j}(c)=0$ and $\lim _{c \rightarrow-\infty} 1 / F_{j}(c)=0$.

p07: For $c \neq 0, \operatorname{sign}(c) . S_{c}\left(s_{j}\right) \downarrow$ as $j \uparrow$.

For $c>0, R_{c}$ prefers better moves to worse ones; for $c<0, R_{c}$ prefers worse moves to better ones.

As the arguments are intuitively straightforward, all theorems' proofs are deferred to Appendix A.

Theorem 2. For $T_{j, c} \neq 0$ and $k>j$, let $r_{j, k, c} \equiv T_{k, \mathrm{c}} / T_{j, c}$. Then $r_{j, k, c} \downarrow$ as $c \uparrow$, and $\lim _{c \rightarrow \infty} r_{j, k, c}=0$.

In particular, $T_{k, c}=r_{D, k, c} \cdot T_{D, c}$ and for $k>D, T_{k, \mathrm{c}}=0$ or $T_{k, \mathrm{c}} \downarrow$ as $c \uparrow$ with $\lim _{c \rightarrow \infty} T_{k, \mathrm{c}}=0$.

Theorem 3. $S_{c}$ properties $p 03-p 06 \Rightarrow$ the spectrum properties $p 01-p 02$ of $R_{c}$.

Note that $p 07$ and $p 08$ are not needed to prove the key Theorem 3 . However, $p 07$ attributes a consistent degree of skill to the $R_{c}$, and $p 08$ reasonably considers positions less differentiable as depth increases.

Theorem 4. As $c \rightarrow \infty, R_{c}$ 's $T_{D, c} \rightarrow 1, \mathrm{E}_{c}[s \mid P] \rightarrow D$ and $\mathrm{E}_{c}[\operatorname{val}(s) \mid P] \rightarrow \operatorname{val}\left(s_{D}\right)$. Thus, $\lim _{c \rightarrow \infty} R_{c} \equiv R_{\infty}$ and $\lim _{c \rightarrow-\infty} R_{c} \equiv R_{-\infty}$ exist, and are respectively the infallible and anti-infallible players.

\section{AN EXAMPLE PREFERENCE FUNCTION}

Let $S F_{c}($ win,$d)=(d+\kappa)^{-c}, S F_{c}($ loss,$d)=,\lambda .(d+\kappa)^{c}$ for some $\kappa>0, d_{W}>n_{W}$ and $d_{L}>n_{L}$, with $\lambda$ determined by: $\left(d_{W}+\kappa\right)^{-c}=S_{c}\left(\operatorname{win}, d_{W}\right)=S_{c}($ draw $)=S_{c}\left(\right.$ loss,$\left.d_{L}\right)=\lambda \cdot\left(d_{L}+\kappa\right)^{c}$, i.e. $\lambda=\left[\left(d_{W}+\kappa\right) \cdot\left(d_{L}+\kappa\right)\right]^{-c}$.

$\kappa>0$ is not only necessary to ensure property p03 but also appropriate. Wins on the move are missed, and even cases of resigning instead are on record (Krabbé, 2002) ${ }^{6}$. Also, positions where the loser is forced to make a capture are possible. In Figures 1 to $3, \kappa=1$ but in Sections 7 et seq, $S F_{c}$ is used with $\kappa=0^{+}$, i.e., arbitrarily small.

Theorem 5. The function $S F_{c}$ satisfies Preference Function Properties $p 03-p 08$.

Figure 1 shows the Preference Function $S F_{c}$ for KQKR (DTC) and wtm with $c=\kappa=1$. Parameters $d_{W}$ and $d_{L}$ are set to the minimum values possible (32 and 4) for KQKR, wtm. They could however have been arbitrarily large, making the likelihood of $R_{c}$ conceding theoretical value arbitrarily small. Figure 2 shows the Preference Function for KRKQ and wtm, and Figure 3 shows the effect on $S_{c}\left(s_{0}\right)-S_{c}\left(s_{31}\right)$ of increasing $c$.

\section{BASIC USES OF REPS}

The assumption of a fallible opponent enables a player to distinguish between metric-optimal ${ }^{7} \mathrm{moves}^{\text {In }}$ Browne-BELLE position BB2-22b, the defender Black has a choice between DTC-optimals Rf7 and Rf6. Assuming White plays only win-preserving moves, Figure 4 shows that 22 ... Rf7 is preferred regardless of $c$. Similar clear choices are available in positions BB1-09b and BB1-13b, namely Re1 in both cases.

\footnotetext{
${ }^{6}$ Sanguineti-Najdorf (1956) 3K1Q2/8/2p1kpbp/3p2p1/3Pr1P1/8/8/8 b: 1... Res. ?? \{1... Rxg4" 2. Qe7+ Kf5 $\left.{ }^{\circ}+\right\}$. Ortega-Etcheverry (1963), 8/pp2Q2p/1bp3pk/4Pp1n/1P6/4qN2/P2nB1PP/4BK2 w: 1. Res.?? \{1. Bxd2" Qxd2 1-0\}. Dekhanov-Yusupov (1981), 8/pp6/q5pp/1Q2Np1k/5P2/P5PK/1Pr4P/8 w: 1. Res.?? \{1. g4+" fxg4² 2. Nxg4+" g5 1-0\}. Glek-Lazarev (1997), 5Rk1/5rpp/p3Q3/1p1p4/4qp2/7P/5BP1/7K b: 1... Res.?? \{1. ... Kxf8 2. Bc5+ Re7" 0-1\}.

${ }^{7}$ The chosen metric is DTC. Figures 4-7 graph expected (DTC) depth after White's next move against competence $c$. Black seeks to maximise this depth. Table 1 lists all positions referenced here.
} 


\begin{tabular}{|cc|cc|cc|}
\hline \multicolumn{2}{|c|}{ Position } & \multicolumn{2}{|c|}{ Position } & \multicolumn{2}{c|}{ Position } \\
BB1-01w & wKa8Qa5/bKf6Re8 & BB1-13b & wKd5Qf6/bKg4Re8 & BB2-22b & wKe4Qe3/bKg4Rf5 \\
BB2-01w & wKc8Qd8/bKc3Rc4 & BB2-10b & wKc5Qg6/bKe3Rf4 & BB2-24b & wKe4Qg3/bKh5Rf7 \\
BB1-09b & wKd6Qd3/bKf4Re4 & BB2-12b & wKc5Qc3/bKe2Ra4 & J1-1b & wKe3Qc5/bKf5Ra6 \\
\hline
\end{tabular}

Table 1: KQKR positions.

At position BB2-10b, Black has a similar decision between DTC-optimals Ra4 and Re4. As Figure 5 shows, no move dominates across the whole range of $c$ : Ra4 is preferable against poor players but Re4 is significantly better for $c>6$. A probability distribution for the opponent's competence would therefore be useful: Game 1, move $8 \mathrm{~b}$, is a similar scenario. Section 8 below describes how opponent competence can be assessed.

The ranking of Black's options for $c=0$ is determined by the average depth of White's choices; as $c \rightarrow \infty$ it is determined by the profile of White's best responses to Black's moves. Thus, it is not surprising that the two scenarios shown in Figures 4 and 5 can arise.

For position BB2-12b, Black has a choice between DTC-optimals Re4, Rf4, Rg4 or Rh4. The moves Rf4, Re4 and $\operatorname{Rg} 4$ are in turn best, respectively, for $c \in[0,16), c \in[16,35]$ and $c>35$ : Rh4 is never preferred.

For position BB2-24b, Black has a choice between DTC-optimals Rb7, Rf6 and Rf8, q.v. Figure 6. The move Rf6 just dominates Rf8 but is only best for the range $c \in(6,26.5)$. Rb7 is best against both the zero-skill random mover and the near-infallible player.

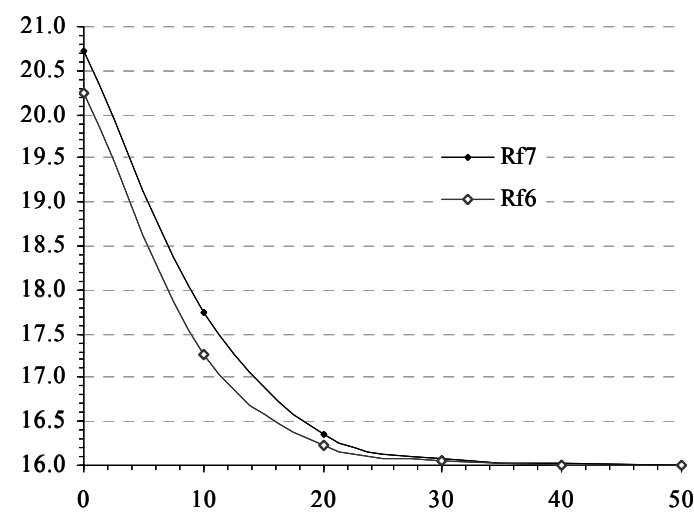

Figure 4: BB2-22b, Rf7 dominates Rf6.

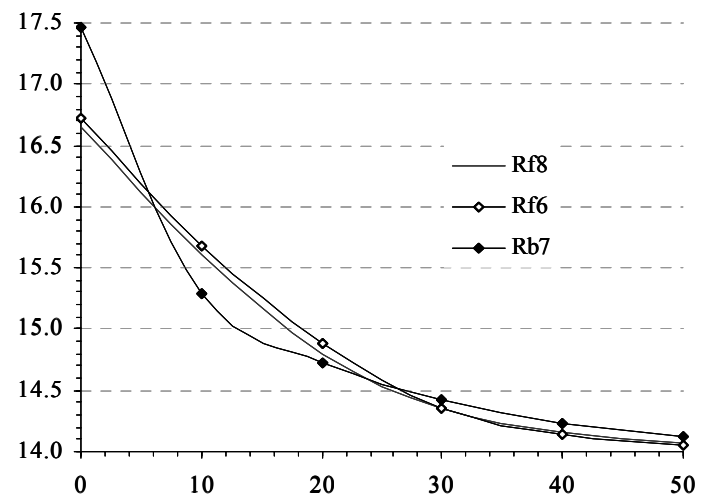

Figure 6: BB2-24b; Rb7, Rf6 ... and Rb7 best.

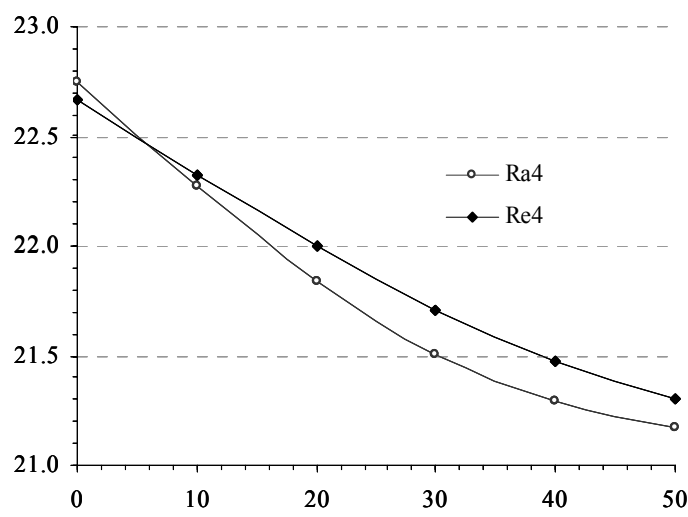

Figure 5: BB2-10b, Ra4 best ... then Re4.

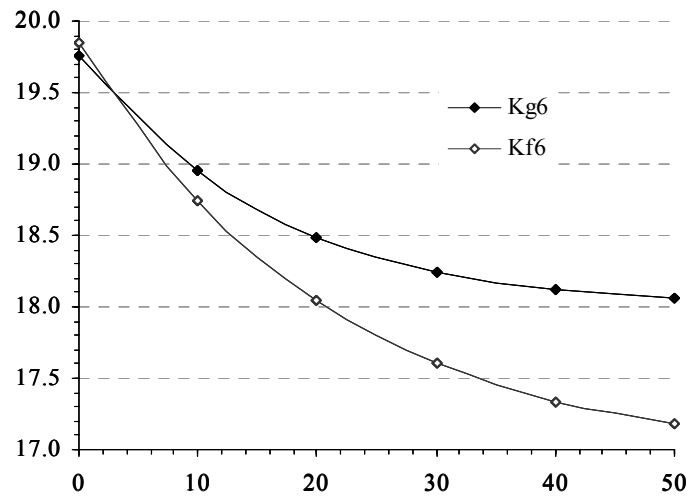

Figure 7: J1-1b; speculative Kf6 only for $c<2.5$.

Finally, we recall Jansen's (1993) suggestion that DTC-sub-optimal moves should also be considered: he illustrated this with another KQKR position, J1-1b. Certainly the move Kf6 which sacrifices one move in depth is at some stage better but, as Figure 7 shows, this is only for $R_{c}$ with $c<2.7$. 
Speculative play has greater potential in the deeper endgames and/or faster playing conditions when fallible opponents are likely to have less competence and lower apparent competence factors $c$. Certainly, there have been some remarkable blunders under time-pressure in blitz and rapid-play, and even in games at classical time-controls.

\section{IDENTIFYING COMPETENCE LEVELS}

Perhaps the earliest demonstration of an infallible player was in two KQKR games (Fenner, 1979; Jansen, 1992a; Levy and Newborn, 1991), cf. Appendix B. GM Walter Browne sportingly volunteered to play Ken Thompson's BELLE. The chess engine, defending, had Thompson's newly calculated KQKR DTC EGT and apparently chose at random between equi-DTC-optimal moves.

The two initial positions, BB1-01w and BB2-01w, are maxDTC positions with DTC $=31$. BELLE claimed a 50move draw in the first game but Browne, after a further bout of exhaustive preparation assisted by the computer's analysis of the first encounter, won exactly on move 50 in the second game. It is intriguing to speculate as to whether BELLE could have squeezed out a second draw. A question worth addressing then is whether BELLE could have set bigger problems for Browne by calculating which DTC-optimal move to choose, or even by playing DTC-suboptimal moves (Jansen, 1993).

Here we propose a way in which BELLE might have developed a probability distribution for Browne's competence $c$. In the same way, an infallible observer can assess two players in a game when both are fallible.

Let us suppose that BELLE had made a conventional, neutral, 'know nothing' initial assumption about Browne's competence level $c$, for example ${ }^{8}$ that $c$ was equally likely to be any integer in $[0,50]$. Because the move of the model's $\left\{R_{c}\right\}$ are chosen independently of previous moves, the initial probabilities, $1 / 51$, could then be adjusted on the basis of observed moves by the following rule of Bayesian inference:

$$
\text { Posterior Prob }[c] \propto \text { Prior Prob }[c] \text {.Prob[observed move } \mid c]
$$

and then $\Sigma_{c} \operatorname{Prob}[c]$.(Expected depth $\left.\mid c\right)$ could be calculated for each move option.

This computation, also reasonably assuming Browne would never choose a drawing or losing move, shows that his competence profiles in the two games are remarkably similar. The first fourteen moves elevate expected $c$, $\mathrm{E}[c]=\Sigma_{i} i$.Prob $[i]$ towards the maximum possible in the model before a few sub-optimal moves bring it sharply down. The effect of the specific move options is occasionally clear. For example, the apparent competence $c$ is unchanged after move 1 as both options are optimal. Note also that after moves 35-39 and 46-50, Browne's $c$ is lower in Game 2 than in the extended Game 1, even though more progress has been made.

On moves 20 to 22 and $32^{+}$of the second game, Browne progressed where he had stalled in game 1. Even so, the final $\mathrm{E}[c]$ values for each game were similar, q.v. Figure 8. Browne conceded DTC depth as follows:

game 1: moves 6w $(+1 / 1), 17 \mathrm{w}(+3 / 4), 18 \mathrm{w}(+2 / 6), 19 \mathrm{w}(+1 / 7), 20 \mathrm{w}(+1 / 8), 21 \mathrm{w}(+2 / 10), 22 \mathrm{w}(+1 / 11)$, 26w $(+2 / 13), 31 \mathrm{w}(+1 / 14), 32 \mathrm{w}(+4 / 18), 33 \mathrm{w}(+2 / 20) \ldots 40 \mathrm{w}(+3 / 23), 41 \mathrm{w}(+2 / 25), 42 \mathrm{w}(+2 / 27)$

game 2: moves 6w $(+1 / 1), 16 \mathrm{w}(+1 / 2), 17 \mathrm{w}(+3 / 5), 19 \mathrm{w}(+3 / 8), 26 \mathrm{w}(+1 / 9), 27 \mathrm{w}(+2 / 11), 28 \mathrm{w}(+2 / 13)$, $30 \mathrm{w}(+1 / 14), 32 \mathrm{w}(+1 / 15), 33 \mathrm{w}(+1 / 16), 35 \mathrm{w}(+2 / 18), 44 \mathrm{w}(+1 / 19)$

BELLE had equi-optimal choices in Game 2 at Table 1's moves 10b, 12b, 22b and 24b, all before the decisive winning cycle of moves 34 to 46, but chose correctly only on move 22b. Assuming that Browne was roughly equivalent to player $R_{20}$, the opponent model shows a further benefit of $\sim 0.26$ moves for the best choices $^{9}$, so perhaps BELLE might have got a second draw. With $c>17$ throughout, it seems unlikely that speculative play choosing sub-optimal moves would have been justified.

In general, a set of fallible players, of which the set $\left\{R_{c}\right\}$ is one example, may be incorporated into a 'PrOM' Probabilistic Opponent-Modelling Strategy (Donkers, Uiterwijk, and Van den Herik, 2001).

\footnotetext{
${ }^{8}$ The range, granularity and probability distribution of the initial assumption certainly affect subsequent inferences. Results here by Andrist with an initial $c=0(1) 50$ differ slightly, as expected, from the original data for $c=0(10) 50$.

${ }^{9}$ For the first game, the further benefit would have been $\sim 0.71$ moves.
} 


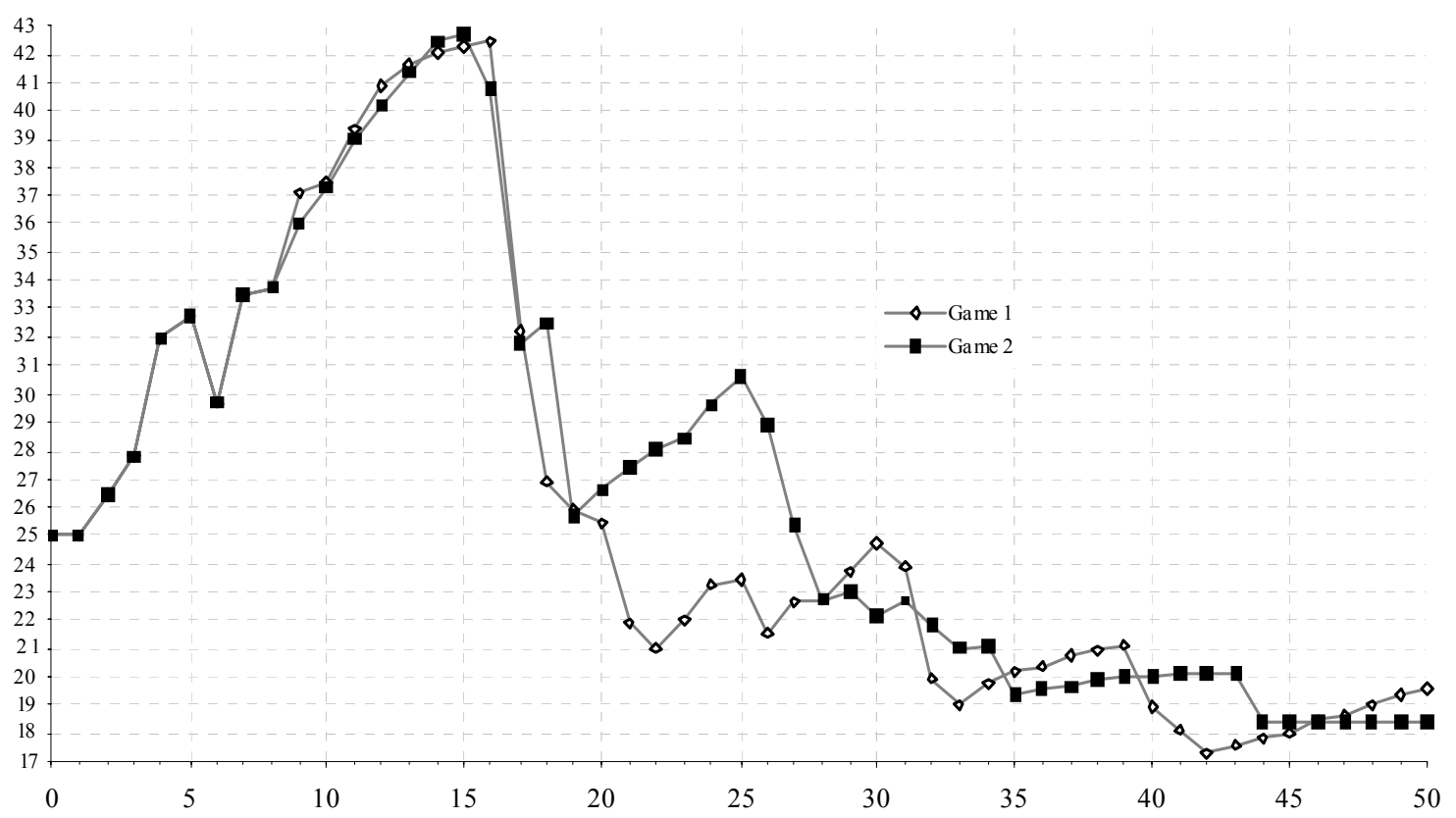

Figure 8: A competency measure $c$ of Walter Browne's play during the two KQKR games.

\section{EXPLOITING REPs}

Below we provide six variants of how to use Reference Players in practice.

\subsection{Endgame Practice Partners}

The current time-regimes, e.g., Professional Chess $\left(40 / 2^{\circ}, 20 / 1^{\circ}\right.$, All/15'+30"m), involve faster rates of play to a finish. Good endgame technique is therefore increasingly important. The fallible players $R_{c}$ would make ideal practice partners, being available, tireless, house-trained, uncritical and non-deterministic in behaviour. They can comment on moves played, be set manually or automatically to a level of difficulty $c$, and give you even more help to win with $c$ set negative. These benefits recommend them as an adjunct to existing chess engines.

Taking the concept of the fallible player beyond the endgame with an EGT, a game-engine may, in general, be able to offer a number of comparable options evaluated on the same basis. If so, an $R_{c}$ player could be defined to choose its moves stochastically rather than always choosing the apparently optimal move.

\subsection{Assessing Player Competence}

The Bayesian inference process may be used to advantage during a game or in later analysis to identify the apparent endgame competence of players. This opportunity also suggests an 'endgame competition' in which chess-engines play without endgame tables and are scored on their apparent competence.

\subsection{Changing the Game Result}

The most significant use of the $R_{c}$ is perhaps to change the game score by differentiating between positions. Attacker can pressure fallible defender and equally, defender can resist fallible attacker (Levy 1987, 1991; Nunn, 2002; Schaeffer 1991, 1997), a scenario surprisingly obviated by the rules of the Kasparov -V- DEEP JUNIOR match. Note that, as usual, the estimated values of positions may be aggregated back to the current position, in this case factored by the calculated probabilities of them occurring rather than minimaxed. Experiments with, e.g., KRBKR will show whether the defender can be effectively backed into 'hard to defend' territory. 


\subsection{Avoiding or Promoting Draw Claims}

Haworth $(2000,2001)$ establishes the DTR, Depth by The Rule, metric as necessary and sufficient for best avoiding $k$-move draw claims, whether $k=50$ or not. An opponent playing to the DTM or DTC metrics is fallible, and may be induced into conceding depth in DTR terms if identified as DTM/DTC-oriented. Even without this information, if the attacker or defender assess the opponent's apparent competence as in Section 8 and Subsection 9.2 above, this will inform their choice of equi-optimal or even sub-optimal moves.

\subsection{Monte-Carlo Simulation}

From a position $P$, games $R_{c}-R_{\infty}$ and $R_{\infty}-R_{c}$ may be played out to demonstrate how well $R_{c}$ can respectively progress the win or put up a defence, and what apparent competence $c$ the $R_{c}$ exhibit. The same data can be compared with the theoretical 'aggregate' results of Theorem 6 below. Matches $R_{c}-R_{c}$ may determine whether a game is more easily won or lost, and how much an attacker can pressure a defender in a drawn position.

\subsection{Assessing Endgame Difficulty}

Here, we derive aggregate characteristics of an endgame as a whole rather than examining play from individual positions. The finite-state model is extended to a Markov model of endgame play.

After possible weighting of moves, we have seen that $R_{c}$ moves from position $P$ in state $s_{i}$ to state $s_{j}$ with probability $T_{j, c}$. Let $R_{c}$ 's average $s_{i} \rightarrow s_{j}$ transition probability across the endgame be $m_{i, j, c}$ giving state-transition matrix $\mathbf{M}_{\mathbf{c}}=\left[m_{i, j, c}\right]$. This Markov model is admittedly somewhat conditioned by the choices of $d_{W}$ and $d_{L}$, move-weighting and parameters such as $S F\left(s_{i}\right)$ 's $\kappa$. There are two matrices $\mathbf{M} \mathbf{W}_{\mathbf{c}}$ and $\mathbf{M B}_{\mathbf{c}}$ for, White's and Black's moves respectively, and now we require that White's wins are represented by states 0 to $n_{W}$, wtm or $\mathrm{btm}$. We may directly prove that, if value-changing moves are excluded, $\operatorname{Pr}\left[R_{c}\right.$ securing the win $]=1$ for any finite $c$. It is not true, as Jansen (1992a, p128, Fig. 12) perhaps suggests, that a low-skill fallible player hits a 'no progress' barrier and cannot win. Even players with low, even negative, $c$ have a long enough run of luck eventually, despite expecting to lose ground at most depths.

Theorem 6. With $\mathbf{M}=\mathbf{M W}_{\mathbf{w}} \cdot \mathbf{M} \mathbf{W}_{\mathbf{b}}$, the state-probability vectors satisfy $\mathbf{p}_{\mathbf{n}}{ }^{\mathrm{T}}=\mathbf{p}_{\mathbf{0}}{ }^{\mathrm{T}} \cdot \mathbf{M}^{\mathbf{n}}$, and $\mathbf{p}_{\mathbf{n}}{ }^{\mathrm{T}}$ converges to a steady state solution $\mathbf{p}^{\mathrm{T}}=\mathbf{p}^{\mathrm{T}}$.M. The expected depth after $n$ moves from the initial $\mathbf{p}_{0}$ is $\sum_{i} \operatorname{depth}\left(s_{i}\right) \cdot p_{n, i}$. The expected length $\underline{\mathbf{L}}$ of the game $R_{w}-R_{b}$ starting at $s_{i}$ is $l_{i}$ as defined by $(\mathbf{M}-\mathbf{I}) \cdot \underline{\mathbf{L}}=\underline{\mathbf{-}}$ with $l_{0}=l_{n s-1}=0$.

Theorem 7. Let $\mathrm{E}_{\mathrm{c}}[s \mid \mathbf{p}]$ be the state expected by $R_{c}$ after a move from a position $P$ with a state-probability vector $\mathbf{p}$. Then, if $c 1<c 2, \mathrm{E}_{\mathrm{c} 2}[s \mid \mathbf{p}] \leq \mathrm{E}_{\mathrm{cl} 1}[s \mid \mathbf{p}]$ and $\mathrm{E}_{\mathrm{c} 2}[\operatorname{val}(s) \mid \mathbf{p}] \geq \mathrm{E}_{\mathrm{c} 1}[\operatorname{val}(s) \mid \mathbf{p}]$.

The proof follows directly from Theorem 3 . However, it need not be true that $\mathrm{E}_{\mathrm{c} 2}[s] \leq \mathrm{E}_{\mathrm{cl} 1}[s]$ after $n>1$ moves from $P$. A stronger player can be repulsed sooner than a weaker player, leaving the latter briefly 'ahead'.

\section{SUMMARY}

A number of questions arise as to how best to attack or defend against a fallible opponent. How best can a result be created or expedited? How competent is the opponent? This contribution has defined a spectrum of REPs $R_{c}$ whose use enables these and other questions to be addressed. Some reasonably tractable computations have been defined which could assess the aggregate difficulty of at least 3 to 5 man endgames.

The REPs are now analysing past play by carbon and silicon players, testing the sensitivity of the results to the initial assumptions of competence. Their Monte-Carlo results are being compared with the Markov model theory of the endgame as a whole. REP-model sensitivity to parameters such as $d_{W}, d_{L}$ and $\kappa$ is being addressed.

My thanks go first to John Tamplin (2001) for providing many Nalimov-compatible 3-5-man pawnless endgame DTC EGTs, including KQKR used here. Then I would like to thank Billy Stewart for guidance on Markov systems and the referees for their positive feedback. Finally, and not least, I acknowledge Rafael Andrist (2002) who has incorporated the REPs in WILHELM. This has enabled the finer-grain ' $c=0(1) 50$ ' Browne-BELLE analysis and the calculation of some Markov matrices. During his experiments, the original REP concept was generalised from its original form as presented at the $7^{\text {th }}$ Computer Olympiad Workshop, Maastricht (2002). 


\section{REFERENCES}

Andrist, R. (2002). http://www.geocities.com/rba_schach2000/.

Donkers, H.H.L.M., Uiterwijk, J.W.H.M. and Herik, H.J. van den. (2001). Probabilistic opponent-model search. Information Sciences, Vol. 135, Nos. 3-4, pp. 123-149. ISSN 1069-0115.

Fenner, C.J. (1979). Computer Chess, News about the North American Computer Chess Championship. The British Chess Magazine, Vol. 99, No. 5, pp. 193-200. ISSN 0007-0440.

Haworth, G.M ${ }^{\mathrm{c}}$ C. (2000). Strategies for Constrained Optimisation. ICGA Journal, Vol. 23, No. 1, pp. 9-20.

Haworth, G.Mㄷ. (2001). Depth by The Rule. ICGA Journal, Vol. 24, No. 3, p. 160.

Jansen, P.J. (1992a). KQKR: Awareness of a Fallible Opponent. ICCA Journal, Vol. 15, No. 3, pp. 111-131. ISSN 1389-6911.

Jansen, P.J. (1992b). KQKR: Assessing the Utility of Heuristics. ICCA Journal, Vol. 15, No. 4, pp. 179-191.

Jansen, P.J. (1993). KQKR: Speculatively Thwarting a Human Opponent. ICCA Journal, Vol. 16, No. 1, pp. 317.

Krabbé, T. (2002). http://www.xs4all.nl/ timkr/chess2/resigntxt.htm. "The Ultimate Blunder".

Levy, D.N.L. (1987). Improving the Performance of Endgame Databases. ICCA Journal, Vol. 10, No. 4, pp. 191-192.

Levy, D.N.L. (1991). First among equals. ICCA Journal, Vol. 14, No. 3, p. 142.

Levy, D. and Newborn, M. (1991). How Computers Play Chess, esp. pp. 144-148.

Nunn, J. (2002). Secrets of Pawnless Ending, $2^{\text {nd }}$ Edition, esp. pp. 9, 49, 173, 300 and 330. Gambit. ISBN 19019-8365-X

Schaeffer, J. (1991). Checkers: a Preview of What will Happen to Chess? ICCA Journal, Vol. 71, No. 2, pp. 71-78.

Schaeffer, J. (1997). One Jump Ahead: Challenging Human Supremacy in Checkers, esp. p. 375. SpringerVerlag, New York, N.Y. ISBN 0-3879-4930-5.

Tamplin, J. (2001). Private communication of some pawnless Nalimov-compatible DTC EGTs.

\section{APPENDIX A: PROOFS OF THEOREMS}

Theorem 1. With the following definitions of properties $p 06-p 07,\{p 03-p 05, q 06-q 09\} \Leftrightarrow\{p 03-p 07\}$ :

p06: $F_{j}(c)=S_{c}\left(s_{j+1}\right) / S_{c}\left(s_{j}\right) \downarrow$ as $c \uparrow: \lim _{c \rightarrow \infty} F_{j}(c)=0$ and $\lim _{c \rightarrow-\infty} 1 / F_{j}(c)=0$. p07: For $c \neq 0, \operatorname{sign}(c) . S_{c}\left(s_{j}\right) \downarrow$ as $j \uparrow$.

Proof. $S_{c}($ win,$d) / S_{c}($ win,$d+1)=S_{c}\left(s_{d}\right) / S_{c}\left(s_{d+1}\right)$ : $S_{c}$ lloss, $\left.d+1\right) / S_{c}($ loss,$d)=S_{c}\left(s_{j}\right) / S_{c}\left(s_{j+1}\right)$ for $j=n_{W}+2+n_{L}-d$. $p 05 \wedge q 06 \wedge q 07 \Rightarrow \forall j, F_{j}(c)=S_{c}\left(s_{j+1}\right) / S_{c}\left(s_{j}\right) \downarrow$ as $c \uparrow$ and $\lim _{c \rightarrow \infty(-\infty)} F_{j}(c)=0(\infty) \Rightarrow p 06$. $p 05 \wedge q 08 \wedge q 09 \Rightarrow$ for $c \neq 0, \operatorname{sign}(c) . S_{c}\left(s_{j}\right) \downarrow$ as $j \uparrow \Rightarrow p 07$.

Further, $p 06 \Rightarrow q 06 \wedge q 07$ and $p 07 \Rightarrow q 08 \wedge q 09 . \therefore\{p 03-p 05, q 06-q 09\} \Leftrightarrow\{p 03-p 07\}$.

Theorem 2. For $T_{j, c} \neq 0$ and $k>j, r_{j, k, c} \equiv T_{k, c} / T_{j, c} \downarrow$ as $c \uparrow$, and $\lim _{c \rightarrow \infty} r_{j, k, c}=0$. In particular, $T_{k, c}=r_{D, k, c} \cdot T_{D, c}$ and for $k>D, T_{k, \mathrm{c}}=0$ or $T_{k, \mathrm{c}} \downarrow$ as $c \uparrow$ with $\lim _{c \rightarrow \infty} T_{k, \mathrm{c}}=0$.

Proof. $r_{j, k, c}=T_{k, c} / T_{j, c}=\left[\omega_{c} \cdot w_{k} \cdot S_{c}\left(s_{k}\right)\right] /\left[\omega_{c} \cdot w_{j} \cdot S_{c}\left(s_{j}\right)\right]=\left(w_{k} / w_{j}\right) . \prod F_{j}(\mathrm{c}) \times \ldots \times F_{k-1}(\mathrm{c})$. p06 $\Rightarrow F_{j}(c) \downarrow$ as $c \uparrow$ and $\lim _{c \rightarrow \infty} F_{j}(c)=0$. Therefore, for $k>j, r_{j, k, c}$ has the same properties. As $T_{j, c}=0$ for $j<D$ and $T_{D, c} \neq 0, T_{k, c}=r_{D, k, c} \cdot T_{D, c}$ and $\lim _{c \rightarrow \infty} T_{k, \mathrm{c}}=0$.for all $k>D$.

Theorem 3. $S_{c}$ properties $p 03-p 06 \Rightarrow$ the spectrum properties $p 01-p 02$ of $R_{c}$.

Proof. $p 06 \Rightarrow \lim _{c \rightarrow \infty} S_{c}\left(s_{j+1}\right) / S_{c}\left(s_{j}\right) \equiv \lim _{c \rightarrow \infty} F_{j}(c)=0 \Rightarrow \lim _{c \rightarrow \infty} R_{c}=R_{\infty}$, the infallible player. Similarly, $\lim _{c \rightarrow-\infty} S_{c}\left(s_{j+1}\right) / S_{c}\left(s_{j}\right) \equiv \lim _{c \rightarrow-\infty} F_{j}(c)=\infty \Rightarrow \lim _{c \rightarrow-\infty} R_{c}=R_{-\infty}$, the anti-infallible player. $p 04 \Rightarrow R_{0}$ is the random player for which all moves are equally likely. Thus, $p 04 \wedge p 06 \Rightarrow p 01$.

Let $T_{j, c}=0$ for $j<D$ and $T_{D, c} \neq 0$. $\sum_{j} T_{j, c 2}=1=T_{D, c 2} \cdot \sum_{j=D} r_{D, j, c 2}=T_{D, c 1} . \sum_{j=D} r_{D, j, c 1}$. Further, $r_{D, j, c 2} \leq r_{D, j, c l} \Rightarrow T_{D, c 2} \geq T_{D, c 1}$. If $T_{D, c 2}>T_{D, c 1}$ then there is an $E$ such that $T_{j, c 2}>T_{j, c 1}$ for $j<E$ but $T_{E, c 2}<T_{E, c 1}$. $\therefore$ for $j \geq E, T_{j, c}=r_{E j, c} . T_{E, c}$ and $r_{E, j, c 2} \leq r_{E, j, c l}$. Therefore, $T_{j, c 2}=r_{E, j, c 2} \cdot T_{E, c 2}<r_{E, j, c l} . T_{E, c l}=T_{j, c l}$ for $j \geq E$. $\sum_{j} T_{j, c 2}=1=\sum_{j=1 \ldots E-1} T_{j, c 2}+\sum_{j=E} T_{j, c 2}=\sum_{j=1 \ldots E-1} T_{j, c 1}+\sum_{j=E} T_{j, c 1}$ 
Therefore $\sum_{j=D \ldots E-1}\left(T_{j, c 2}-T_{j, c l}\right)=\sum_{j=E}\left(T_{j, c 1}-T_{j, c 2}\right)$

But $\left(T_{j, c 2}-T_{j, c l}\right) \geq 0$ for $j=D$ to $E-1$ and $\left(T_{j, c 1}-T_{j, c 2}\right) \geq 0$, for $j=E$ to $n s$.

$\therefore \sum_{j=1 \ldots E-1} j .\left(T_{j, c 2}-T_{j, c l}\right) \leq \sum_{j=E \ldots \ldots} j .\left(T_{j, c l}-T_{j, c 2}\right)$ and $\sum_{j} j \cdot T_{j, c 2} \leq \sum_{j} j . T_{j, c l} \Rightarrow \mathrm{E}_{\mathrm{c} 2}[s \mid P] \leq \mathrm{E}_{\mathrm{cl}}[s \mid P]$.

As $i<j \Rightarrow \operatorname{val}\left(s_{i}\right) \geq \operatorname{val}\left(s_{j}\right), \sum_{j=1 \ldots E-1} \operatorname{val}(j) .\left(T_{j, c 2}-T_{j, c l}\right) \geq \sum_{j=E} \operatorname{val}(j) .\left(T_{j, c l}-T_{j, c 2}\right)$

$\therefore \sum_{j} \operatorname{val}\left(s_{j}\right) \cdot T_{j, c 2} \geq \sum_{j} \operatorname{val}\left(s_{j}\right) \cdot T_{j, c l} \Rightarrow \mathrm{E}_{\mathrm{c} 2}[\operatorname{val}(s) \mid P] \geq \mathrm{E}_{\mathrm{c} 1}[\operatorname{val}(s) \mid P]$.

Thus, property $p 02$ is ensured.

Theorem 4. As $c \rightarrow \infty, R_{c}$ 's $T_{D, c} \rightarrow 1, \mathrm{E}_{c}[s \mid P] \rightarrow D$ and $\mathrm{E}_{c}[\operatorname{val}(s) \mid P] \rightarrow \operatorname{val}\left(s_{D}\right)$. Thus, $\lim _{c \rightarrow \infty} R_{c} \equiv R_{\infty}$ and $\lim _{c \rightarrow-\infty} R_{c} \equiv R_{-\infty}$ exist, and are respectively the infallible and anti-infallible players.

Proof. $T_{j, c}=0$ for $j \leq D$ and $T_{D, c} \neq 0 . \mathrm{E}_{c}[$ state $]=\mathrm{E}_{c}[s]=\Sigma_{k=0} k \cdot T_{k, c}=\Sigma_{k=D} k \cdot T_{k, c}$.

From Theorem 2, we have $T_{k, c}=r_{D, k, c} . T_{D, c} . \therefore \lim _{c \rightarrow \infty} T_{k, c}=0$ for $k>D$ and $\lim _{c \rightarrow \infty} T_{D, c}=1$.

Thus, we may define $\lim _{c \rightarrow \infty} R_{c} \equiv R_{\infty}$ with $T_{D, \infty}=1$ and $T_{k, \infty}=0$ for $k \neq D$.

$\therefore \mathrm{E}_{c}[s \mid P]=D . T_{D, c}+\sum_{k=D+1} . k \cdot r_{D, k, c} \cdot T_{D, c} \Rightarrow \lim _{c \rightarrow \infty} \mathrm{E}_{c}[s]=D$.

Similarly, $\mathrm{E}_{c}[\operatorname{val}(s) \mid P]=\Sigma_{k=D} \operatorname{val}\left(s_{i}\right) \cdot T_{k, c}=\operatorname{val}\left(s_{D}\right) \cdot T_{D, c}+\Sigma_{k=D+1} \operatorname{val}\left(s_{k}\right) \cdot T_{k, c}$.

Therefore, $\lim _{c \rightarrow \infty} \mathrm{E}_{c}[\operatorname{val}(s)]=\operatorname{val}\left(s_{D}\right)$.

Similarly, we may show that $\lim _{c \rightarrow-\infty} R_{c} \equiv R_{-\infty}$.

Theorem 5. The function $S F_{c}$ satisfies Preference Function Properties $p 03-p 08$.

Proof. $S F_{c}($ win,$d)=(d+\kappa)^{-c}$ and $S F_{c}($ loss,$d)=,\lambda .(d+\kappa)^{c}$ with constants $\lambda, \kappa>0$.

p03:: $\kappa>0 \Rightarrow d+\kappa>0 \Rightarrow \forall$ real $c,(d+\kappa)^{c}>0$ and finite $\Rightarrow S F_{c}(s)>0$ and finite $\Rightarrow p 03$.

p04:: $S F_{0}($ loss,$d)=(d+\kappa)^{0}=1, S F_{0}($ draw $)=S F_{0}\left(\right.$ loss,$\left.d_{L}\right)=1$ and $S F_{0}($ win,$d)=(d+\kappa)^{-0}=1 \Rightarrow p 04$.

p05:: true by definition of $S F_{c}$.

p06:: For $j=0$ to $n_{W}, F_{j}(c)=S F_{c}\left(S_{j+1}\right) / S F_{c}\left(S_{j}\right)=(j+\kappa)^{c} /(j+1+\kappa)^{c}=\left[1-(j+1+\kappa)^{-1}\right]^{c}=a^{c}$ with $a \in(0,1)$.

$\therefore \lim _{c \rightarrow \infty} F_{j}(c)=0$ and $\mathrm{d} F_{j} / \mathrm{d} c=\ln _{\mathrm{e}}\left[1-(j+1+\kappa)^{-1}\right] .\left[1-(j+1+\kappa)^{-1}\right]^{c}<0$.

For $j=n_{W}+1$ to $n s-2, F_{j}(c)=S F_{c}\left(s_{j+1}\right) / S F_{c}\left(s_{j}\right)=S F_{c}($ loss,$n s-j-1) / S F_{c}($ loss,$n s-j)$

$\therefore F_{j}(c)=\lambda .(n s-j-1+\kappa)^{c} / \lambda .(n s-j+\kappa)^{c}=\left[1-(n s-j+\kappa)^{-1}\right]^{c} \in(0,1)$

$\therefore \lim _{c \rightarrow \infty} F_{j}(c)=0$ and $\mathrm{d} F_{j} / \mathrm{d} c=\ln _{\mathrm{e}}\left[1-(n s-j+\kappa)^{-1}\right] .\left[1-(n s-j+\kappa)^{-1}\right]^{c}<0$.

$\therefore F_{j}(c)=S F_{c}\left(S_{j+1}\right) / S F_{c}\left(S_{j}\right)$ is a decreasing function of $c$ and $\lim _{c \rightarrow \infty} F_{j}(c)=0$.

p07:: For $j=0$ to $n_{W}+1, f(j) \equiv \operatorname{sign}(c) \cdot \mathrm{S}_{\mathrm{c}}\left(s_{j}\right)=\operatorname{sign}(c) \cdot(j+\kappa)^{-c}$ and $d f / d j=-c \cdot \operatorname{sign}(c) \cdot(j+\kappa)^{-c-1}$

For $j=n_{W}+1$ to $n s, f(j) \equiv S_{c}\left(s_{j}\right)=\operatorname{sign}(c) \cdot \lambda \cdot(n s-j+\kappa)^{c}$ and $d f / d j=-c \cdot \operatorname{sign}(c) \cdot \lambda \cdot(n s-j+\kappa)^{c-1}$

Therefore, for $c \neq 0, \operatorname{sign}(c) \cdot S_{c}\left(s_{j}\right)$ is a decreasing function of $j$ : for $\mathrm{c}=0$, it is a constant.

p08:: $W_{c}(j)=S_{c}($ win,$j) / S_{c}($ win,$j+1)=(j+1+\kappa)^{c} /(j+\kappa)^{c}=\left[1+(j+\kappa)^{-1}\right]^{c} \rightarrow 1$ as $j \rightarrow \infty$.

$\therefore d W / d j=\alpha^{2}\left[c .(j+\kappa)^{c} . c .(j+1+\kappa)^{c-1}-(j+1+\kappa)^{c} . c .(j+\kappa)^{c-1}\right]=\beta^{2} \cdot c \cdot[(j+\kappa)-(j+1+\kappa)]=-\beta^{2} . c$.

$\therefore$ For $c>(<) 0, W_{c}(j)=S_{c}($ win,$j) / S_{c}($ win,$j) \downarrow(\uparrow)$ as $j \uparrow$ with $\lim _{j \rightarrow \infty} W_{c}(j)=1$.

$L_{c}(j)=S_{c}($ loss,$j+1) / S_{c}($ loss,$j)=\lambda \cdot(j+1+\kappa)^{c} / \lambda \cdot(j+\kappa)^{c} \equiv W_{c}(j)$.

$\therefore$ For $c>(<) 0, L_{c}(j)=S_{c}($ loss,$j+1) / S_{c}($ loss,$j) \downarrow(\uparrow)$ as $j \uparrow$ with $\lim _{j \rightarrow \infty} W_{c}(j)=1$.

Theorem 6. With $\mathbf{M}=\mathbf{M W}_{\mathbf{w}} \cdot \mathbf{M} \mathbf{W}_{\mathbf{b}}$, the state-probability vectors satisfy $\mathbf{p}_{\mathbf{n}}{ }^{\mathrm{T}}=\mathbf{p}_{\mathbf{0}}{ }^{\mathrm{T}} \cdot \mathbf{M}^{\mathbf{n}}$, and $\mathbf{p}_{\mathbf{n}}{ }^{\mathrm{T}}$ converges to a steady state solution $\mathbf{p}^{\mathrm{T}}=\mathbf{p}^{\mathrm{T}}$.M. The expected depth after $n$ moves from the initial $\mathbf{p}_{0}$ is $\sum_{i} \operatorname{depth}\left(s_{i}\right) \cdot p_{n, i}$. The expected length $\underline{\mathbf{L}}$ of the game $R_{w}-R_{b}$ starting at $s_{i}$ is $l_{i}$ as defined by $(\mathbf{M}-\mathbf{I}) \cdot \underline{\mathbf{L}}=\underline{\mathbf{-}}$ with $l_{0}=l_{n s-1}=0$.

Proof. Let $p_{n, j}=\operatorname{Prob}[$ system in state $j$ after move $n]$. Let $\mathbf{M}=\left[m_{i, j}\right]$.

Then $p_{n, j}=\sum_{i} p_{n-1, i} \cdot m_{i, j}$, i.e. $\mathbf{p}_{\mathbf{n}}{ }^{\mathrm{T}}=\mathbf{p}_{\mathbf{n}-1}{ }^{\mathrm{T}} \cdot \mathbf{M}$ and therefore $\mathbf{p}_{\mathbf{n}}{ }^{\mathrm{T}}=\mathbf{p}_{\mathbf{0}}{ }^{\mathrm{T}} \cdot \mathbf{M}^{\mathbf{n}}$.

The probability that the system-state is fixed $\uparrow$ to a limit which may be proved here to be 1 .

Therefore, $\mathbf{p}_{\mathbf{n}}{ }^{\mathrm{T}} \rightarrow \mathbf{p}^{\mathrm{T}}$ which satisfies $\mathbf{p}^{\mathrm{T}}=\mathbf{p}^{\mathrm{T}} \cdot \mathbf{M}$ and is a left-eigenvector for the eigenvalue $\lambda=1$.

Let $l_{s}=\mathrm{E}\left[R_{w}-R_{b}\right.$ game-length, starting from state $\left.s\right] . l_{0}=l_{n s-1}=0$ as states 0 and $n s-1$ are finished games.

Then $l_{i}=1+\sum_{j} m_{i, j} \cdot l_{j} \Rightarrow-1=\sum_{j \neq i} m_{i, j} \cdot l_{j}+\left(m_{i, i}-1\right) \cdot l_{i} \Rightarrow(\mathbf{M}-\mathbf{I}) \cdot \underline{\mathbf{L}}=\underline{\mathbf{- 1}}$.

Theorem 7. Let $\mathrm{E}_{\mathrm{c}}[s \mid \mathbf{p}]$ be the state expected by $R_{c}$ after a move from a position $P$ with a state-probability vector $\mathbf{p}$. Then, if $c 1<c 2, \mathrm{E}_{\mathrm{c} 2}[s \mid \mathbf{p}] \leq \mathrm{E}_{\mathrm{c} 1}[s \mid \mathbf{p}]$ and $\mathrm{E}_{\mathrm{c} 2}[\operatorname{val}(s) \mid \mathbf{p}] \geq \mathrm{E}_{\mathrm{c} 1}[\operatorname{val}(s) \mid \mathbf{p}]$.

Proof. Let $\mathbf{p}=\sum_{j} p_{j} \underline{\underline{\mathbf{u}}}_{\mathbf{j}}$ where $\underline{\mathbf{u}}_{\mathbf{j}}$ indicates that the system is in state $s_{j}$.

Let the number of positions $P$ in state $s_{j}$ be $N P_{j}$. Then $\mathrm{E}_{c}\left[s \mid \underline{\mathbf{u}}_{j}\right]=\mathrm{E}_{c}\left[s \mid \sum_{P \in \text { state } j} P\right] / N P_{j}$.

But $\mathrm{E}_{c}[s \mid \mathbf{p}]=\mathrm{E}_{c}\left[s \mid \sum_{j} p_{j} \cdot \underline{\mathbf{u}}_{\mathbf{j}}\right]=\sum_{j} p_{j} . \mathrm{E}_{c}\left[s \mid \underline{\mathbf{u}}_{j}\right]$, i.e. $\mathrm{E}_{c}[s \mid \mathbf{p}]$ is a linear function $\mathrm{R}^{n s} \rightarrow \mathrm{R}$.

$\therefore \mathrm{E}_{c}[s \mid \mathbf{p}]=\sum_{j} p_{j} \mathrm{E}_{c}\left[s \mid \sum_{P \in \text { state } j} P\right] / N P_{j \cdot}$. But $\mathrm{E}_{c 2}[s \mid P] \leq \mathrm{E}_{c l}[s \mid P]$ from Theorem 3 .

Therefore $\sum_{j} p_{j} \mathrm{E}_{c 2}\left[s \mid \sum_{P \in \text { state } j} P\right] / N P_{j} \leq \sum_{j} p_{j} \mathrm{E}_{c l}\left[s \mid \sum_{P \in \text { state } j} P\right] / N P_{j}$, i.e. $\mathrm{E}_{\mathrm{c} 2}[s \mid \mathbf{p}] \leq \mathrm{E}_{\mathrm{cl}}[s \mid \mathbf{p}]$.

Similarly, $\mathrm{E}_{c}[\operatorname{val}(s) \mid \mathbf{p}]$ is a linear function $\mathrm{R}^{n s} \rightarrow \mathrm{R}$ and $\mathrm{E}_{\mathrm{c} 2}[\operatorname{val}(s) \mid \mathbf{p}] \geq \mathrm{E}_{\mathrm{c} 1}[\operatorname{val}(s) \mid \mathbf{p}]$. 


\section{APPENDIX B: THE BROWNE-BELLE GAMES}

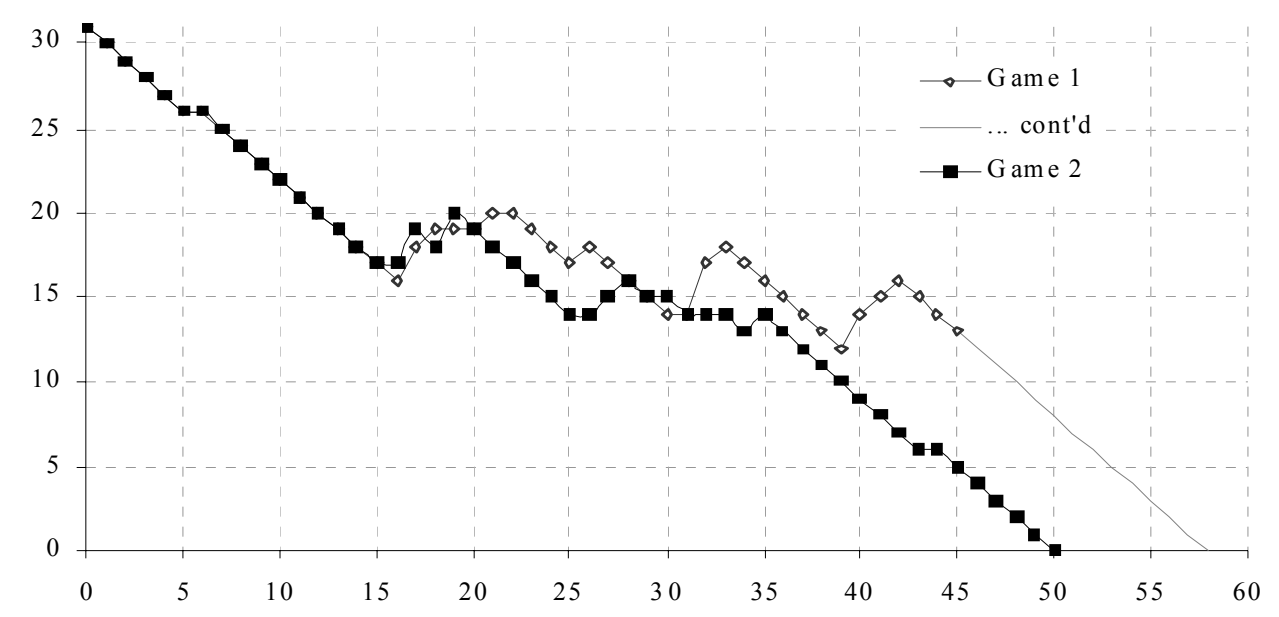

Figure 9: The two Browne-BELLE games: depth after move $m$.

These games have been annotated with respect to the DTC metric as follows:

$" \equiv$ only value-preserving move, ${ }^{\prime} \equiv$ only optimal move, ${ }^{\circ} \equiv$ only legal move,

$\{+i /+j: \ldots\} \equiv$ move conceding $i$ moves in depth, making a total loss of $j$ moves in depth in the game

[Re4, Rg4, Rh4] $\equiv$ DTC-optimal moves for Black

;: $\equiv$ DTC equi-optimal move preferred by the model of opponent fallibility.

Game 1: $\left\{\right.$ BB1-1w: wKa8Qa5/bKf6Re8+w, 19 ${ }^{\text {th }}$ Dec., 1978; $\left.d c=31\right\}$ 1. Kb7 Re7+' 2. Kc6 Re6+' 3. Kd7 Re7+' 4. Kd8' Re4' 5. Qc5 Re5' 6. Qd4 \{+1/+1: Qc3, Qc4, Qf8+ and Qg1 optimal $\}$ Kf5' 7. Kd7 Re4' 8. Qd3 Kf4 (:) [Ke5] 9. Kd6 Re3 [Re1 (;) 10. Qd4+ Re4' 11. Qf2+ Kg4' 12. Kd5 Re8 [Re7 (;)] 13. Qf6 Re3 [Re1 (;)] 14. Kd4 Rf3' 15. Qg6+ Kf4' 16. Qg2 Ra3' 17. Qc6 $\{+3 /+4:$ Qd2 + , Qe4+ optimal $\}$ Ra1' 18. Qc7+ $\{+2 /+6$ : Qc2'\} Kf5' 19. Qc2+ \{+1/+7: Qc8+'\} Ke6' 20. Qd2 \{+1/+8: Qc4+'\} Ra7 (:) [Ra4+] 21. Qb4 \{+2/+10: Qe1+, Qe2+, Qe3+, Qh6+ optimal $\}$ Re7' 22. Ke4 $\{+1 /+11:$ Qa5, Qb3+, Qc3, Qc5, Qe1+ optimal $\}$ Kf6+' 23. Kf4' Ke6' 24. Qd4 Rf7+' 25. Ke4' Rf6' 26. Qd5+ $\{+2 /+13:$ Qd8' $\}$ Ke7 $^{\circ}$ 27. Ke5' Rh6 [Rg6 (;)] 28. Qb7+ Kd8' 29. Qf7' Rc6 (-) [Ra6] 30. Kd5' Rb6' 31. Kc5 \{+1/+14: Qf4'\} Ra6' 32. Qc4 \{+4/+18: Qd5+'\} Rf6' 33. Qh4 $\{+2 /+20: K d 5 ' . d c=18$ so Black has the draw $\}$ Ke7' 34. Kd5' Kf7' 35. Ke5 Re6+' 36. Kf5' Rd6' 37. Qc4+' Ke7' 38. Ke5' Rh6 (-) [Rg6] 39. Qc7+' Kf8' 40. Kf5 \{+3/+23: Qd7'\} Ke8' 41. Qc1 \{+2/+25: Qe5+'\} Rd6' 42. Qc8+ $\{+2 /+27$ : Qc7' $\}$ Ke7' 43. Qc7+ Rd7' 44. Qc5+' Kd8' 45. Ke6 Rb7' $\{d c=13$ : draw agreed $\}$. 1/2-1/2.

White conceded 27 moves in depth over moves 6, 17-22, 26, 31-33 and 40-42. Black had DTC-optimal choices on moves 8, 9, 12, 13, 20, 27, 29 and 38, and made the 'model' choice on moves 8, 20, 29 and 38.

Game 2: $\left\{\mathbf{B B 2} 2 \mathbf{1 w}\right.$ : wKc8Qd8/bKc3Rc4+w, 30 ${ }^{\text {th }}$ Dec., 1978; $\left.d c=31\right\} \mathbf{1}$. Kb7 Rb4+' 2. Kc6 Rc4+' 3 . Kb5 Rb4+' 4. Ka5' Re4' 5. Qd6 Rd4' 6. Qe5 $\{+1 /+1$ : Qa3+, Qe6, Qf6 and Qh2 optimal $\}$ Kd3' 7. Kb5 Re4' 8. Qf6 Ke3' 9. Kc5 Rf4' 10. Qg6 Ra4 [Re4 (;)] 11. Qg3+ Ke2' 12. Qc3 Rf4 [Re4, Rg4 (;), Rh4] 13. Kd5' Rh4' 14. Qc2+' Ke3' 15. Qd1' Kf2' 16. Qd2+ $\{+1 /+2:$ Qd3'\} Kf3' 17. Qe1 $\{+3 /+5:$ Qd3+'\} Rg4' 18. Qd1+ Kf4' 19. Qe2 \{+3/+8: Qc1+\} Rg5+' 20. Kd4' Rf5' 21. Qe3+ Kg4' 22. Ke4 Rf7 (;) [Rf6] 23. Qg1+' Kh5' 24. Qg3' Rf8 [Rb7 $;$, Rf6] 25. Ke5' Rf7' 26. Ke6 $\{+1 /+9$ : Qd3' $\}$ Rf8' 27. Qa3 $\{+2 /+11:$ Qe5+' $\}$ Rf4' 28. Qh3+ $\{+2 /+13$ : Qg3'\} Kg5' 29. Qg3+ Rg4' 30. Qe5+ \{+1/14: Qe3+'\} Kh4' 31. Qh2+' Kg5 32. Ke5 \{+1/+15: Qd2+'\} Kg6' 33. Qh8 $\{+1 /+16$ : Qc2+ and Qh3 optimal $\}$ Rg5+' 34. Ke6' a pattern repeated at moves 37b, 40b, 43b and 46b\} Rg4' 35. Qg8+ \{+2/+18: Qe5'\} Kh5' 36. Qh7+' Kg5 37. Ke5' Rg3' 38. Qg7+' Kh4' 39. Qh6+' Kg4' 40. Ke4' Rg2' 41. Qg6+' Kh3' 42. Qh5+' Kg3' 43. Ke3' Rg1' 44. Qg5+ \{+1/+19: Qe5', and now White cannot slip again $\}$ Kh2' 45. Qh4+' Kg2 ${ }^{\circ}$ 46. Ke2' Ra1' 47. Qe4+ Kh3' 48. Qh7+' Kg3 [Kg2, Kg4] 49. Qg7+' Kh3 50. Qxa1' \{Just in time! Black resigns.\} 1-0.

White ceded 19 moves in depth over moves 6, 16-17, 19, 26-28, 30, 32-33, 35 and 44. Black had DTC-optimal choices on moves 10, 12, 22 and 24, and made the 'model' choice on move 22 only. 